irritation. The symptoms are of a twofold nature; first, there is inverted peristaltic action, causing constipation and vomiting, and secondly, under the influence of perverted nervous action morbid secretions are thrown out. The indications of treatment are:-1st. To exhaust for a time the excitability of the nerves proceeding from the lower part of the abdomen, so as to prevent their carrying to the spinal cord the morbid impressions which are reflected to the stomach; and this can be done, as physiologists are well aware, by over-stimulating them as by the free application of turpentine epithems. 2nd. To restore the peristaltic action to its natural order, to be accomplished by the use of suitable aperients, given by the mouth or by enemata. 3rd. To remove the uterine or ovarian irritation either by sedatives applied directly to the parts as by suppositories; or where there is evidence of inflammation applying leeches, or caustic, or other appropriate treatment, to its seat; and, when these means fail, and the prostration is so great as to endanger the patient's life, by inducing premature labour.

\title{
(10bituary 嗮otice.
}

\section{ROBERT JOHNS, A.B., M.B., L.K.\& Q.C.P.I., F.R.C.S.I.,} Died 11th May, 1864, aged 49 years.

To the long list of the medical men of Dublin who have recently been cut off from amongst us, we have to add the name of Dr. JoHNs, who died on the 11th of May, after an illness of but a few days' duration. Dr. Johns was an active and energetic practitioner, and was well known to the readers of this Journal by his frequent communications to its pages. He was one of the original members of the Dublin Obstetrical Society, of which, at an early period, he was elected an honorary member, and one of the Honorary Vice-Presidents; and, on hearing of his death, the Council held a special meeting, at which they resolved, as a mark of respect for his memory, to adjourn the general meeting of the Society that had been previously announced. 